\title{
Effect of nickel substitution on thermal properties of $\mathrm{Na}_{0.9} \mathrm{CoO}_{2}$
}

\author{
J MEENA DEVI*, N GAYATHRI ${ }^{\dagger}$, A BHARATHI ${ }^{\dagger}$ and K RAMACHANDRAN \\ School of Physics, Madurai Kamaraj University, Madurai 625 021, India \\ ${ }^{\dagger}$ Materials Science Division, Indira Gandhi Centre for Atomic Research, Kalpakkam 603 102, India
}

MS received 6 November 2006; revised 22 June 2007

\begin{abstract}
We report on the effect of nickel substitution in $\mathrm{Na}_{0.9} \mathrm{CoO}_{2}$ by examining their thermal properties at room temperature. Experimental results indicate that thermoelectric efficiency is enhanced upon nickel substitution in sodium cobaltate.
\end{abstract}

Keywords. Sodium cobaltate; thermoelectric power; photoacoustics; thermal conductivity; figure of merit.

\section{Introduction}

The widespread interest in sodium cobalt oxide is mainly due to its unique combination of high thermopower with metallic transport properties (Terasaki et al 1997), which makes it one of the most promising systems for power application in comparison with conventional thermoelectric material composed of doped semiconductor. The recent discovery of superconductivity, $T_{\mathrm{c}} \sim 5 \mathrm{~K}$ (Takada et al $2003)$, in the hydrated cobaltate, $\mathrm{Na}_{x} \mathrm{CoO}_{2} \cdot y \mathrm{H}_{2} \mathrm{O}(x \sim 0 \cdot 35$, $y \sim 1 \cdot 3)$, has triggered further attention to this layered transition-metal oxide. The physical properties in these $\mathrm{Na}_{x} \mathrm{CoO}_{2}$ materials strongly depend on sodium concentration and phase change (Maw et al 2004) of this material ranges from that of a paramagnetic metal $(x \sim 0 \cdot 3)$ to a charge-ordered insulator (at $x=0 \cdot 5$ ) and to a Curie-Weiss metal $(x \sim 0 \cdot 7)$. Sodium cobaltate has a hexagonal layered structure with a space group, $P 6_{3} / m m c$, consisting of twodimensional layers of $\mathrm{CoO}_{2}$ alternating with sodium ions in two partially occupied sites. $\mathrm{CoO}_{2}$ layer is a triangular network of edge sharing oxygen octahedra and this common structural unit is responsible for the electric conduction and large thermopower.

Electronic specific heat coefficient $(\gamma)$ of this transition-metal oxide (Ando et al 1999) is found to be at about $48 \mathrm{~mJ} / \mathrm{mol} \mathrm{K}^{2}$, which is relatively large when compared to simple metals where $\gamma$ is usually a few $\mathrm{mJ} / \mathrm{molK}^{2}$, which indicates that this system is a strongly correlated system. The Hall coefficient, $R_{\mathrm{H}}$, behaves (Wang et al 2003a) anomalously at high temperature (T). From 200$500 \mathrm{~K}, R_{\mathrm{H}}$ increases linearly with $T$ with no sign of saturation. This behaviour of $R_{\mathrm{H}}$ provides firm evidence for a strong correlation.

Wang et al (2003b) reported that the thermopower, $Q$, in $\mathrm{Na}_{x} \mathrm{CoO}_{2}(x=0.68)$ is completely suppressed by a 10 -

*Author for correspondence (jmeenadevi@rediffmail.com)
Tesla longitudinal magnetic field. The field suppression of $Q$ constitutes direct evidence for a large spin-entropy term in the Seebeck coefficient.

Large degeneracy of cobalt ions of various spin states (Koshibae et al 2000), together with the strong correlation of $3 d$ electrons, causes large thermopower in cobalt oxides.

A general understanding has been reached that the high thermopower arises because of the spin entropy (Wang et al 2003b) gained in the transport of an electron from low spin $\mathrm{Co}^{3+}$ state to a low spin $\mathrm{Co}^{4+}$ state. The ratio of $\mathrm{Co}^{3+} /$ $\mathrm{Co}^{4+}$ is important in determining the ground state properties of $\mathrm{Na}_{x} \mathrm{CoO}_{2}$ and hence any substitution that changes this ratio will affect the resistivity as well as the magnitude of the thermopower.

In addition to studies with varying $\mathrm{Na}$ stoichiometry, there have been studies (Premila et al 2006) on $\mathrm{CoO}_{2}$ layer doping. Substitution of transition metals like Ni, Mn and Ir in place of cobalt induces a metal-to-insulator transition even for the smallest concentration. Metal-to-insulator transition takes place in sodium nickel cobaltate $\left(\mathrm{Na}_{0.9}\right.$ $\left.\mathrm{Co}_{0.99} \mathrm{Ni}_{0.01} \mathrm{O}_{2}\right)$ at $26 \mathrm{~K}$ while $\mathrm{Na}_{0.9} \mathrm{CoO}_{2}$ remains metallic even at low temperature. In the present investigation, thermal properties of two samples, sodium cobaltate $\left(\mathrm{Na}_{0.9} \mathrm{CoO}_{2}\right)$ and sodium nickel cobaltate $\left(\mathrm{Na}_{0.9} \mathrm{Co}_{0.99} \mathrm{Ni}_{0.01} \mathrm{O}_{2}\right)$, at room temperature are studied experimentally to investigate the effect of nickel doping in sodium cobaltate.

\section{Synthesis}

The polycrystalline samples used in this study were prepared by conventional solid state reaction technique using $\mathrm{Na}_{2} \mathrm{CO}_{3}, \mathrm{Co}_{3} \mathrm{O}_{4}$ and $\mathrm{NiO}$ as starting materials. The raw materials were first calcined at $873 \mathrm{~K}$ for $12 \mathrm{~h}$ and subsequently pelletized and sintered at $1123 \mathrm{~K}$ for $18 \mathrm{~h}$. To improve the grain connectivity which can influence the resistivity and thermoelectric properties, the samples were reground, pelletized and heat treated at $1123 \mathrm{~K}$ in flowing 
oxygen for a further $20 \mathrm{~h}$. X-ray diffraction measurements were carried out in a STOE diffractometer operating with $\mathrm{CuK} \alpha$ radiation. The $\mathrm{Ni}$ content in the sample was determined using a Philips ESEMXL30 scanning electron microscope equipped with an EDAX attachment.

$\mathrm{X}$-ray diffraction measurements (figure 1) indicate that the samples crystallize in the hexagonal space group, $\mathrm{P}_{3} / m m c$. No additional peaks are seen due to addition of $\mathrm{Ni}$ because nickel is substituted in the cobalt site forming a homogeneous mixture as well as because X-ray scattering factor is nearly same for cobalt and nickel as they occupy the nearby position in the group table. The lattice parameters for $0 \% \mathrm{Ni}$ are $a=2.8302 \pm 0.0009 \AA$ and $b=10.901 \pm$ $0.004 \AA$. The lattice parameters for $1 \% \mathrm{Ni}$ are $a=$ $2 \cdot 8338 \pm 0.0001 \AA$ and $c=10 \cdot 852 \pm 0.007 \AA$. Nickel doping slightly reduced the $c$-axis lattice parameter and there is no change in the $a$-axis lattice parameter. Densities (d) calculated from the lattice parameters for sodium cobaltate and sodium nickel cobaltate are $4.90 \mathrm{~g} / \mathrm{cm}^{3}$ and $4.92 \mathrm{~g} / \mathrm{cm}^{3}$, respectively.

\section{Electrical resistivity}

The room temperature resistivity is measured by four-probe method. On 1\% nickel doping, resistivity $(\rho)$ decreased from 8-3.5 $\mathrm{m} \Omega \mathrm{cm}$ (table 1 ).

\section{Thermoelectric power}

In the experimental arrangement the sample was sandwiched between two copper blocks using tripod spring arrangement. The $\Delta T$ achieved with the help of a maganin heater placed below one of the $\mathrm{Cu}$ blocks was measured using a differential ( $\mathrm{Au}: 0.07 \% \mathrm{Fe}, \mathrm{Chromel})$ thermocouple attached to the blocks and referenced to the cold end of the sample (measured by a cernox thermometer). The $\Delta V$ was measured using two $\mathrm{Cu}$ wires welded to the $\mathrm{Cu}$ blocks very close to the region of contact with the sample.

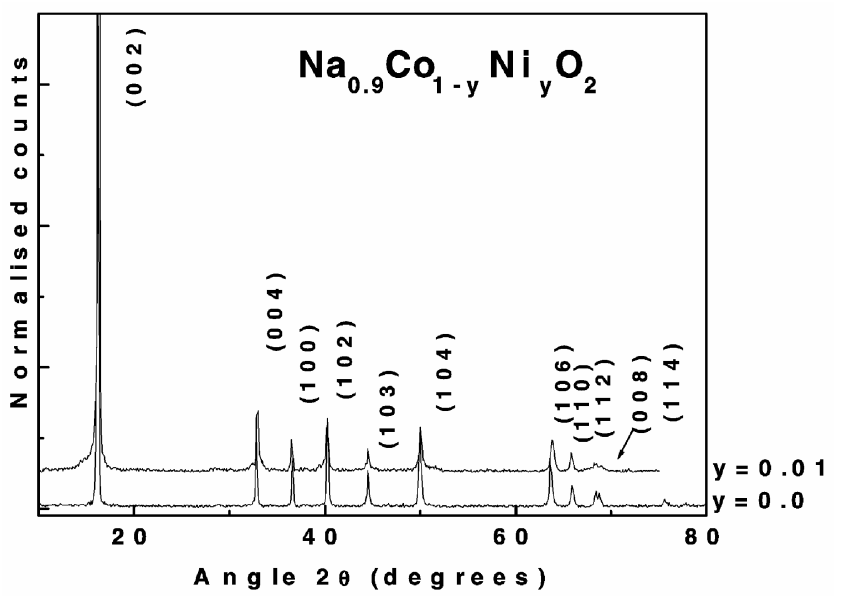

Figure 1. XRD pattern of sodium cobaltate.
In a typical measurement, after temperature stabilization, the $\Delta T$ heater was switched on and the $\Delta V$ and $\Delta T$ were recorded simultaneously until $\Delta T$ incremented to $\sim 1 \mathrm{~K}$. In this regime a linear behaviour was ensured. The thermopower of the sample is found by $S_{\text {sample }}=S_{\mathrm{Cu}}-(\Delta V$ vs $\Delta T)_{\text {slope }}$ and is given in table 1 . On $1 \%$ nickel doping thermoelectric power $(Q)$ increased from $120-140 \mu \mathrm{V} / \mathrm{K}$ (table 1) and thermal conductivity is measured here by photoacoustic technique to correlate the above results.

\section{Photoacoustics}

Photoacoustic (PA) technique is a simple and reliable nondestructive technique for the characterization of thermal, optical and structural properties of the material. This technique has attracted considerable attention because of the ease with which optical spectra of substance normally difficult to examine by conventional spectroscopic method can be obtained. (Photoacoustic spectroscopy is different from conventional techniques chiefly in that sample under analysis is examined not through the subsequent detection of photons but rather through a direct measure of the energy absorbed by the sample as a result of its interaction with a photon beam.) When a modulated light is absorbed by the sample located in a sealed cell (PA cell), the nonradiative deexcitation process of the absorbed light generates modulated transfer of heat to the surface of the sample. This modulated thermal gradient produces pressure waves in the gas inside the cell and the microphone coupled to the cell can detect such oscillations (Rosencwaig and Gersho 1976). The resulting PA signal depends not only on the amount of heat generated in the sample but also on how the heat diffuses through the sample.

\subsection{Experimental}

The present photoacoustic (PA) spectrometer (figure 2) consists of a $450 \mathrm{~W}$ Xenon lamp (Jobin Yvon), an electromechanical chopper (Model number PAR 650), a lock-inamplifier (Model Perkin Elmer 7225 DSP) and PA cell made of stainless steel. White light from Xenon lamp is mechanically chopped by an electromechanical chopper (Model number PAR 650) and focused onto the sample placed inside the PA cell. The PA signal generated is detected by a microphone and it is fed to a lock-in-amplifier where readings are recorded.

\subsection{Thermal conductivity}

For measuring the thermal conductivity of the sample at room temperature photoacoustic signal is recorded as a function of chopping frequency and is shown in figure 3. Thermal diffusivity (Madhusoodanan and Philip 1988) is calculated from the characteristic frequency of the sample 
Table 1. Transport properties of sodium cobaltate at room temperature.

\begin{tabular}{lcc}
\hline Property & $\mathrm{Na}_{0 \cdot 9} \mathrm{CoO}_{2}$ & $\mathrm{Na}_{0 \cdot 9} \mathrm{Co}_{0 \cdot 99} \mathrm{Ni}_{0 \cdot 01} \mathrm{O}_{2}$ \\
\hline Resistivity $(\rho)(\mathrm{m} \Omega \mathrm{cm})$ & 8 & $3 \cdot 5$ \\
Thermo power $(Q)(\mu \mathrm{V} / \mathrm{K})$ & 120 & 140 \\
Thermal diffusivity $(\alpha)\left(\times 10^{-6} \mathrm{~m}^{2} / \mathrm{s}\right)$ & $6 \cdot 61$ & $4 \cdot 57$ \\
Thermal conductivity $(k)(\mathrm{W} / \mathrm{mK})$ & $21 \cdot 12$ & 14.70 \\
Lattice thermal conductivity $\left(k_{\mathrm{ph}}\right)(\mathrm{W} / \mathrm{mK})$ & $21 \cdot 03$ & 14.49 \\
Electronic thermal conductivity $\left(k_{\mathrm{e}}\right)(\mathrm{W} / \mathrm{mK})$ & $0 \cdot 09$ & $0 \cdot 21$ \\
Figure of merit $(\mathrm{ZT})$ & 0.0026 & 0.01 \\
\hline
\end{tabular}

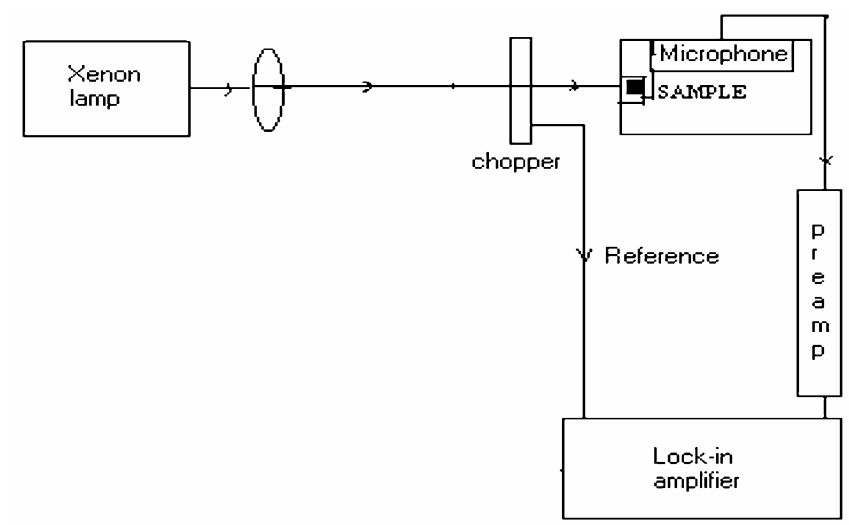

Figure 2. Schematic of photoacoustic spectrometer.

(the characteristic frequency is defined as the frequency at which the sample goes from thermally thick region to thermally thin region and where the slope change is observed in the graph). If $f_{\mathrm{c}}$ is the characteristic frequency of the sample of thickness, $l$, then the thermal diffusivity $(\alpha)$ can be calculated from the relation,

$$
\alpha=f_{\mathrm{c}} l^{2} \mathrm{~m}^{2} / \mathrm{s} \text {. }
$$

From $\alpha$, thermal conductivity $(k)$ is deduced by using

$$
k=\alpha d C_{\mathrm{p}} \mathrm{W} / \mathrm{mK} \text {. }
$$

We have used the specific heat capacity $\left(C_{\mathrm{p}}\right)$ values of $\mathrm{Na}_{0.7} \mathrm{CoO}_{2}$ determined by Wooldridge et al (2004) in (2) and thermal conductivity value thus obtained lies nearly in the range of thermal conductivity value $(\sim 16 \mathrm{~W} / \mathrm{mk})$ of $\mathrm{Na}_{0.7} \mathrm{CoO}_{2}$ measured by Maw et al (2004). On nickel doping, thermal conductivity decreased from $21 \cdot 12-14 \cdot 70 \mathrm{~W} / \mathrm{mK}$.

\section{Results and discussion}

Thermoelectric materials should have high thermoelectric power, low resistivity and low thermal conductivity. Even $1 \%$ nickel doping caused drastic changes in electrical resistivity, thermoelectric power and thermal conductivity. Figure-of-merit $(Z)$ value characterizes the thermoelectric materials and its value calculated for the present samples (table 1) reveals the enhancement of thermoelectric conversion efficiency by nickel doping in $\mathrm{Na}_{0.9} \mathrm{CoO}_{2}$.

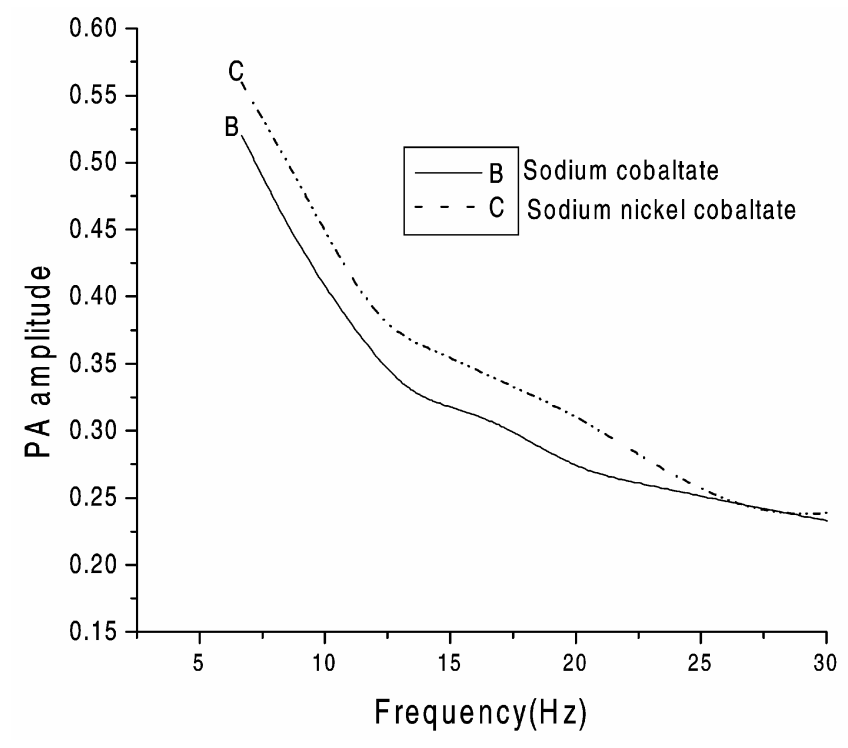

Figure 3. PA amplitude vs chopping frequency of sodium cobaltate samples.

In the high temperature limit, thermopower can be obtained by using Heikes formula (Koshibae et al 2000)

$$
Q \rightarrow \frac{-k_{\mathrm{B}}}{e} \ln \left(\frac{g_{3}}{g_{4}}\left(\frac{n}{1-n}\right)\right),
$$

where $g_{3}$ and $g_{4}$ are the degeneracies of the electronic states in $\mathrm{Co}^{3+}$ and $\mathrm{Co}^{4+}$ ions (low spin), respectively and $n$ the fraction of $\mathrm{Co}^{4+}$ ions. Since Ni (Gayathri et al 2005, 2006) enters the lattice in $4+$, a low spin state, its degeneracy is the same as that of $\mathrm{Co}^{3+}$, whereas the degeneracy of $\mathrm{Co}^{4+}$ is 6 . With nickel substitution, $n$ decreases from a value of 0.1 in the unsubstituted sample to 0.09 in the nickel substituted sample enhancing the thermopower.

Thermal conductivity is one of the basic transport properties that provide useful information on the charge carriers and phonons. In sodium cobalt oxide, $\mathrm{CoO}_{2}$ layer is responsible (Takahata et al 2000) for the electric conduction, whereas the Na layer works only as a charge reservoir to stabilize the crystal structure. Since Na ions randomly occupy $50 \%$ of the regular sites, $\mathrm{Na}$ layer is highly disordered and it looks like a glass for the in-plane phonons. Thus, significant reduction of the thermal con- 
ductivity is likely to occur in the sandwich structure made of the crystalline metallic layers and amorphous insulating layers.

Thermal diffusivity and thermal conductivity of these two samples measured by PA technique show a drastic reduction even for 0.01 concentration of nickel. Thermal conductivity comes from two sources of heat transport. Phonons transport heat and lead to lattice thermal conductivity $\left(k_{\mathrm{ph}}\right)$. The electrons (or holes) also transport heat and lead to electronic thermal conductivity $\left(k_{\mathrm{e}}\right)$. Electronic thermal conductivity is estimated by the Weidemann-Franz law and lattice thermal conductivity is calculated as a difference between total thermal conductivity measured by PA technique and electronic thermal conductivity and these values are shown in table 1 . In both the samples lattice contribution is dominating and electronic contribution is relatively very low. Nickel doping decreased the lattice thermal conductivity from 21.03$14.49 \mathrm{~W} / \mathrm{mK}$. This clearly shows that the reduction in thermal conductivity is due to increase in phonon scattering caused by addition of nickel in the lattice. From their analysis of electronic and thermal conductivity of sodium cobaltate at $280 \mathrm{~K}$, Takahata et al (2000) reported that heat conduction here is mainly determined by phonons.

\section{Conclusions}

To conclude, we have investigated the effect of substitution of $3 d$ element nickel on the thermal properties of $\mathrm{Na}_{0.9} \mathrm{CoO}_{2}$. Our experimental studies at room temperature show favourable and remarkable change in the transport properties resistivity, thermo power, thermal conductivity on $1 \%$ nickel doping in $\mathrm{Na}_{0.9} \mathrm{CoO}_{2}$ which in turn enhances the thermoelectric efficiency. The present observed increase in electrical conductivity on nickel doping may be attributed to slight increase in carrier concentration. Increase in thermoelectric power is due to the increase in spin entropy caused by the substitution of nickel. Thermal conductivity of sodium nickel cobaltate is lowered by the reduction of its lattice thermal conductivity because of the increase in phonon scattering induced by nickel.

\section{References}

Ando Yoichi, Miyamoto N, Segawa Kouji, Kawata T and Terasaki I 1999 Phys. Rev. B60 10580

Gayathri N, Bharathi A and Hariharan Y 2005 cond-mat/ 0510342

Gayathri N, Bharathi A, Sastry V S, Sundar C S and Hariharan Y 2006 Solid State Commun. 138489

Koshibae W, Tsutsui K and Maekawa S 2000 Phys. Rev. B62 6869

Madhusoodanan K N and Philip J 1988 Phys. Status Solidi (a)108 775

Maw Lin Foo, Wang Yayu, Watauchi Satoshi, Zandhergen $\mathrm{H}$ W, Tao He, Cava R J and Ong N P 2004 Phys. Rev. Lett. 92 247001

Premila M, Bharathi A, Gayathri N, Yasodha P, Hariharan Y and Sundar C S 2006 Pramana-J. Phys. 67153

Rosencwaig Allan and Gersho Allan 1976 J. Appl. Phys. 47 64

Takada Kazumori, Sakurai Hiroya, Takayama-Muromuchi Eiji, Izumi Foujiyo, Dilanen Ruben A and Sasaki Takayoshi 2003 Nature $\mathbf{4 2 2} 53$

Takahata K, Iguchi Y, Tanaka D, Ioth T and Terasaki I 2000 Phys. Rev. B61 12551

Terasaki I, Sasago Y and Uchinokura K 1997 Phys. Rev. B56 R12685

Wang Yayu, Nyrissa, Rogada S, Cava R J and Ong N P 2003a cond-mat/0305455

Wang Yayu, Nyrissa, Rogada S, Cava R J and Ong N P 2003b Nature $\mathbf{4 2 3} 425$

Wooldridge J, McK Paul D, Balakrishnan G and Lees M R 2004 cond-mat/0406513 